\title{
Analysis of Combined Pelvic Ring and Acetabulum Fractures for their Optimal Management
}

\section{Senthil Kumar Selvarajan*, Inder Gill, Nikil Shah, Vineet Trivedi, Ahmed Galhoum and Tanya Tradoff}

Department of T and 0 , George Eliot Hospital NHS, UK

*Corresponding Author: Senthil Kumar Selvarajan, Department of T and 0, George

Eliot Hospital NHS, UK.
Received: April 27, 2021

Published: May 20, 2021

(C) All rights are reserved by Senthil Kumar

Selvarajan., et al.

\section{Abstract}

Background: Pelvic fractures carry a huge burden on the current health care system and it has a high morbidity and mortality. The survivors carry a huge burden of complications which can have a lifetime implication, it is therefore imperative to understand the fracture pattern and plan for appropriate care which could be a key in optimal care of these critically injured patient. The classical classification system used for these fractures need to be adapted according to the mechanism of injury and severity of trauma which should also guide in decision making process.

Methods: In this retrospective study all pelvic trauma patients who attended Salford royal NHS foundation from 2015 to 2018 were retrieved then the patterns of fractures and mechanism of injury were analysed and correlated.

Results: Combined pelvic ring and acetabular fractures [5] can be sub-categorised into three groups according to mechanism of injury and fracture patterns:

- Type 1: Road traffic collision causes fracture involving anteroposterior compression (APC) 1, 2, 3 along with Anterior wall $(\mathrm{AW})$, Anterior Column (AC), associated both columns (ABC) variety, lateral compression fractures are rare in RTC, hemodynamic stability is dependent of grade of APC than acetabular fracture pattern and treatment can vary from non-operative to complex surgeries.

- Type 2: Jumpers commonly sustain lateral compression (LC) and ABC pattern and have high Injury severity score (ISS) when compared to RTC, majority of them are hemodynamically unstable and require blood products and more often end up in a broad spectrum of pelvic surgery. Mortality rate is high, and we found nearly $30 \%$ of them die within 24 hours in our study.

- Type 3: Cyclers and pedestrians have an equal incidence of type 1 and type 2 fractures and rarely presents with a combination of type 1 and 2. ISS, blood transfusion and mortality are like type 1 and type 2 combined fractures and treatment must be designed after a thorough understanding of fracture types. Often require complex surgery.

Keywords: Anteroposterior Compression (APC); Anterior Wall (AW); Anterior Column (AC); Associated Both Columns (ABC); Lateral Compression (LC); Injury Severity Score (ISS)

\section{Abbreviations}

ABC: Associated Both Column; AC: Anterior Column; APC: Anteroposterior Compression; AW: Anterior Wall; CT: Computed
Tomography; LC: Lateral Compression; MRI: Magnetic Resonance Imaging; MOI: Mechanism of Injury; MVA: Motor Vehicle Accident; OTA: Orthopaedic Trauma Association; PC: Posterior Column; PW: 
Posterior Wall; RTC: Road Traffic Collision; Mch: Master of Surgery (Latin: Magister Chirurgiae); VS: Vertical Shear; PACS: Picture Archiving and Communication System

\section{Introduction}

Pelvic and acetabular fractures are a significant burden to existing trauma services we have undertaken a retrospective analysis on combined fractures of pelvic ring and acetabulum at Salford royal hospital and to understand in detail the fracture pattern in combined pelvic trauma.

Combined acetabular and pelvic ring fractures still appear to be a maze despite advances in orthopaedic surgery. There are no proper guidelines for treatment of these injuries and evidence in literature is limited owing to the rarity of the fracture. Based on literature review of available articles, it can be said that Injury severity score and mortality rates appear to be looking similar in most of the studies but, fracture pattern in pelvic ring and acetabulum is confusing and not well defined, this also raises a question of selection bias.

Only few studies have highlighted the modalities of operative treatment but has not provided any subjective or objective outcome measures and we also could not find any studies determining the connection between mechanism of injury and fracture pattern.

Bearing in mind the poor surgical outcomes of pelvic ring fractures [6] alone described in numerous literatures, an addition of acetabular fractures has made the situation worse Our hypothesis is the if a clear understanding of the fracture pattern could be achieved, then it would help surgeons in making preoperative planning more clearly and to explain the risks of morbidity forehand to the patients.

\section{Aim of the Study}

The aim of this study is to analyse various mechanism of injury along with fracture pattern and injury severity scores of combined pelvic ring and acetabulum [1,2] fractures which might be used for better perioperative planning.

\section{Research design}

This study was done based on a scientific or a positivist approach. As described by Collins., et al. (2003) [4], this approach is historically the mainstream of medical research.
Our desired outcomes were investigated with a retrospective cohort. All pelvic trauma patients who attended Salford royal NHS foundation from 2015 to 2018 were retrieved from the trauma database of the trust using A\&E coding system.

A retrospective approach was adopted due to the unavailability of an ongoing prospective database for this group of population, secondly when the primary outcome was to investigate the fracture pattern in combined injuries and findings its correlation with demographics distribution, mechanism of injury, blood loss, Injury severity Score, mortality and average length of stay, a retrospective analysis allows for a cost efficient data collection.

Finally, the study did not measure subjective or objective outcomes and therefore a prospective approach $[8,9]$ was not required.

Ethics

The study was registered with Audit and Research committee at Salford royal NHS foundation and an approval was granted to conduct an observational analysis. The study was also registered with the EdgeHill university for the partial fulfilment of MCh (full-form) degree of one of our authors.

Ethical committee approval at Salford royal NHS foundation was applied and since the investigation was only through retrospective analysis of electronic medical documents, the committee did not have any conflicts of interest.

\section{Patients and Methods}

Inclusion criteria:

1. Patients attending to Salford royal A\&E from January 2015 to January 2018 with Combined fractures of pelvic ring and acetabulum.

2. Age 18 to 80 years.

3. Mechanism of injury with high velocity.

4. Patients with CT scan of pelvis.

5. Patients referred from other NHS trust with combined injuries.

\section{Exclusion criteria:}

1. Isolated pelvic ring and isolated acetabulum fractures.

2. Patients with doubtful fracture pattern.

3. Patients with only x rays. 
4. Patients with trivial falls.

5. Osteoporotic fractures.

6. Patients with previous pelvic ring or acetabulum fractures.

7. Patients with associated hip fractures.

\section{Data extraction and analysis}

Data was collected from the pelvic trauma data base of the trust with due permission from the orthopaedic research committee. Analysis was also done by use of electronic patient record and PACS (full-form) and relevant statistical equations.

All patients with combined fractures were analysed from attendance to A\&E to discharge. A\&E records were analysed to determine the mechanism of injury, hemodynamic stability requirement of blood products, and other life-threatening associated injuries.

Radiological records such as x-ray and CT scans [15] were analysed by the primary investigator and the consultant orthopaedic surgeon of pelvic trauma team to describe the pattern of fracture configuration.

Injury severity scores were calculated after the secondary survey, by analysing the injuries the patient had sustained along with relevant investigations.

Modality of treatment such as non-operative and operative were analysed to understand the behaviour of the fracture pattern and other parameters such as average length of stay and mortality were observed to determine the severity of injury.

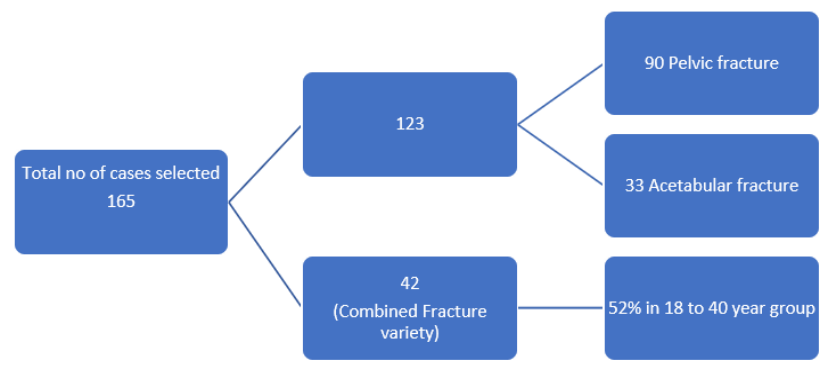

Chart 1: Analysis of the number of cases analysed during this study, 165 number of cases has been analysed of which 42 cases have shown a combined variety of fracture, 123 shows isolated pattern either Pelvic or Acetabular pattern, the interesting part in our study was that $52 \%$ of the combined variety belonged to age group 18 to 40 year group.

\section{Results}

\section{Demographics}

Patients enrolled in the pelvic trauma data base were analysed between January 2015 to January 2018 and a total of 165 patients were evaluated with $\mathrm{x}$ ray and CT scan. We found 42 patients had combined fractures of pelvic ring and acetabulum with an incidence of 20 percent. These findings are suggestive of an increase in incidence compared to Jason J., et al. 2014 quote, 5 to $15.7 \%$.

We analysed the correlation of combined fractures in between age and sex in the given cohort and found its predominance in males of younger age group and an average age of 34.2 (18 to 80). Analysis was done after dividing age into three categories 18 to 40, 40 to 60 , and 60 to 80 and it was noted nearly $52 \%$ of this injury occurred in 18 to 40 age group, with a male dominance of $94 \%$. We also noted similar results in the 40 to 60 and 60 to 80 age groups with male predominance of 85 and $70 \%$ but when compared to the young age group the incidence of combined fractures was low in the 40 to 60 and 60 to 80 age group.

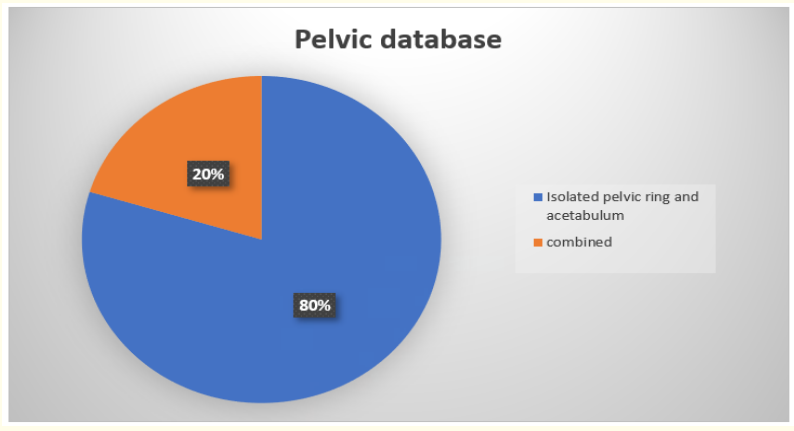

Chart 2: Incidence of combined fracture.

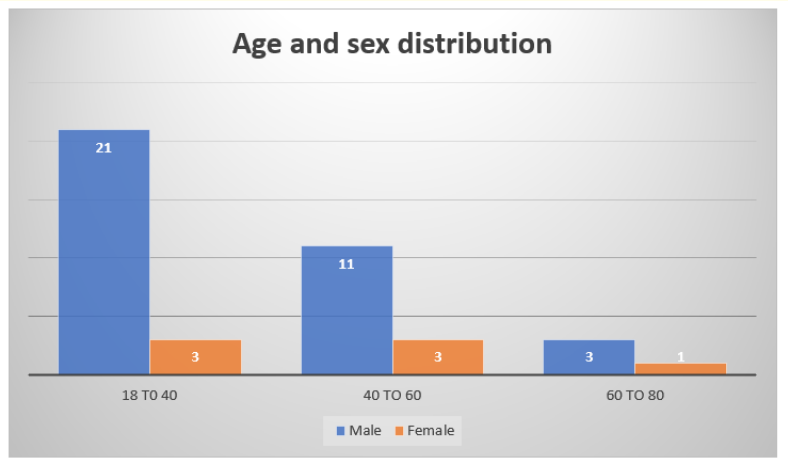

Chart 3: Age and sex distribution. 


\section{Mechanism of injury}

A force which was directed lateral to the body would internally rotate the pelvis and was thought to be the aetiology of combined fractures over decades [20]. We analysed different mechanism and its incidence in between sex. Evaluation was done by categorising the forces into four subsets namely, road traffic collision (RTC), fall from height particularly suicidal jumpers, cyclist/bikers and pedestrians hit by moving vehicle.

In our analysis we found that majority of combined fractures were a direct resultant of RTC contributing to $62 \%$, with males predominantly involved. Cyclers and bikers [22] contributed $17 \%$ of the incidence, on the other hand jumpers with combined fractures were $12 \%$ and pedestrians were $9 \%$ of the cohort. Finally, all the above-mentioned mechanism showed males were more commonly injured than females with an overall incidence of 88\%. Comparing these findings with existing literature, it can be said that there is no single mechanism which leads to combined fractures and also it raises a question on the force vectors which yield to combined injuries do not corelate with fracture configuration as historically believed [24]. This explains the complexity of these injuries.

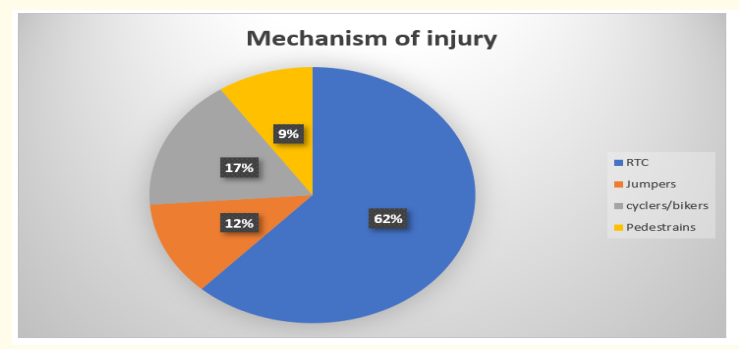

Chart 4: Mechanism of injury.

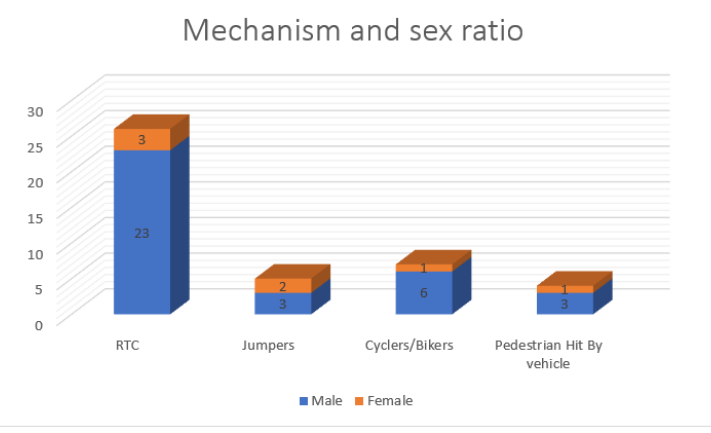

Chart 5: Mechanism of injury in different sex.
We calculated the ISS based on A\&E records and other relevant investigations such as imaging studies which yields to diagnosis and a comparison was made between different mechanism of injury, hemodynamic stability [27] and need for blood products.

We found a mean ISS of 28.6 ranging from (4 to 66) and when compared with different mechanism, it can be said that patients involved in RTC who had an incidence $52 \%$ of combined fractures had a mean ISS of 27.6, whereas jumpers with an incidence of $12 \%$ had a mean ISS of 43.1. This implies that jumpers [29] are more pronounced to associated injuries along with combined fractures. Similarly, comparison when made for cyclers/bikers and pedestrians, we found a mean ISS of 26.2 and 27 respectively.

Hemodynamic stability was assessed through emergency physician assessment notes at the time of arrival to hospital and it was found $62 \%$ of the cohort were detected with low systolic blood pressure and required blood transfusion [30]. Ironically, we observed only 12 out of 26 patients involved in RTC were transfused, in contrast to jumpers were all 5 jumpers in the cohort were activated with massive haemorrhage protocols. Similarly, 6 out of 7 cyclers and 3 out of 4 pedestrians needed blood transfusion. These findings generally suggest that, the severity of injury and requirements of blood transfusion are more frequent in patients were the force is directly applied to the body rather than injuries occurring within a moving vehicle. Moreover 2 patients out of the cohort did not stabilise following transfusion and required emergency embolization [31].

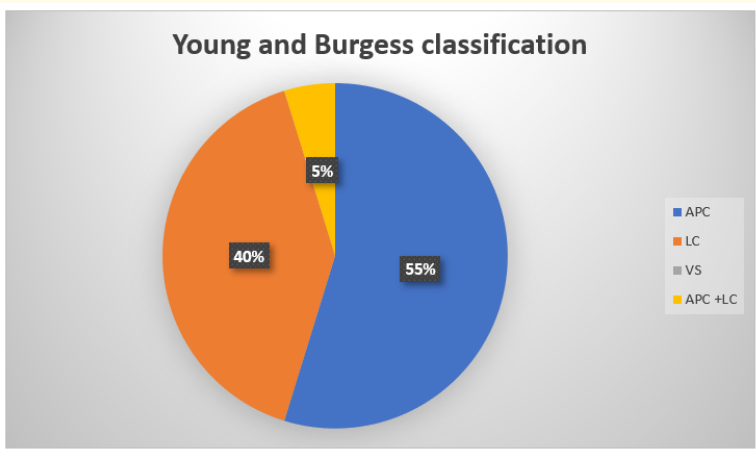

Chart 6: Fracture pattern in pelvic ring.

\section{Discussion}

Pelvic fractures are complex injuries which have mostly been managed in a Level 1 trauma centres or nearly all of them are re- 


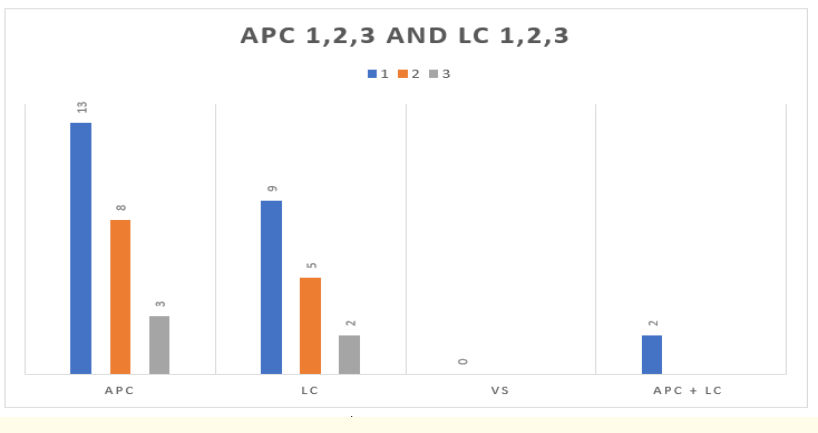

Chart 7: Sub classification of fracture types.

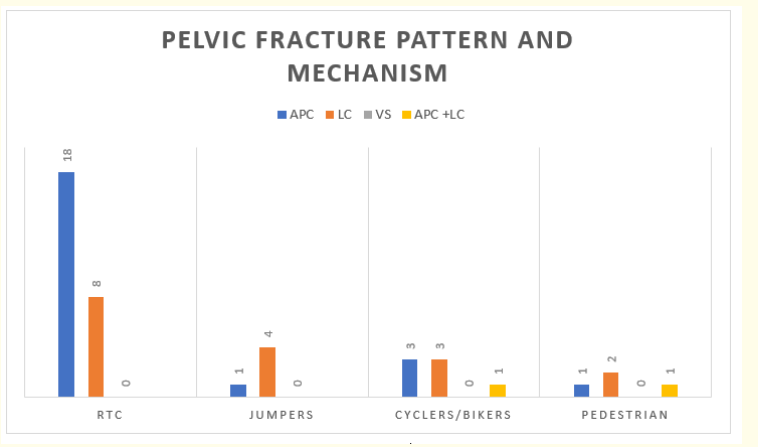

Chart 8: Fracture types and correlation to different MOI.

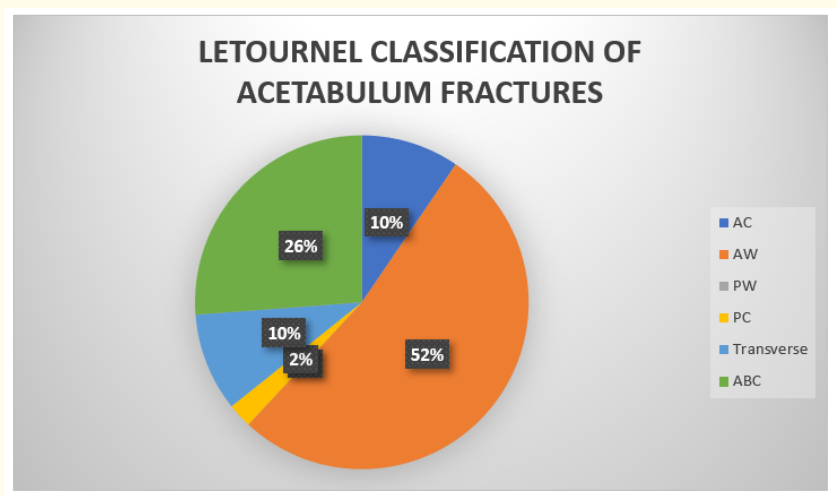

Chart 9: Fracture pattern seen in acetabulum.

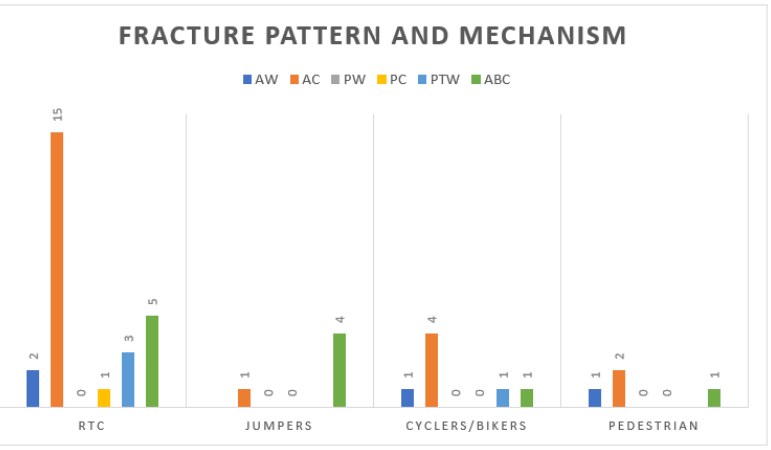

Chart 10: Acetabular fracture pattern in relation different MOI.

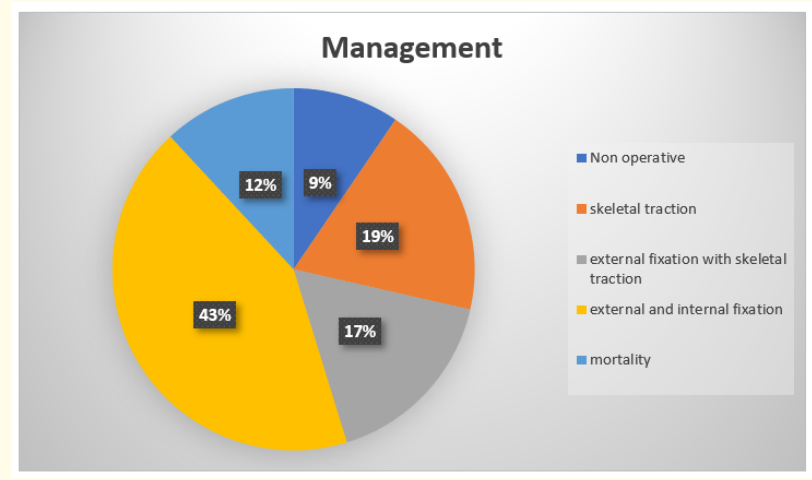

Chart 11: Management of combined fractures.

ferred over there or at least an opinion is sought after initial management to guide the treatment plan, this recommendation which is followed in most unit is as per the National Institute for health and care excellence (NICE) guideline [35] recommendation.

Given the classification system which we have been using so far, it fails to combine the pelvic fracture and acetabular fracture together, unfortunately there are various factors which contributes to this combination of injuries which contributes around $20 \%$ of all pelvic fractures.

The incidence of combined pelvic ring and acetabular fractures is raising, despite advancement in orthopaedic science this entity remains a challenge. The common fracture pattern noted are APC 


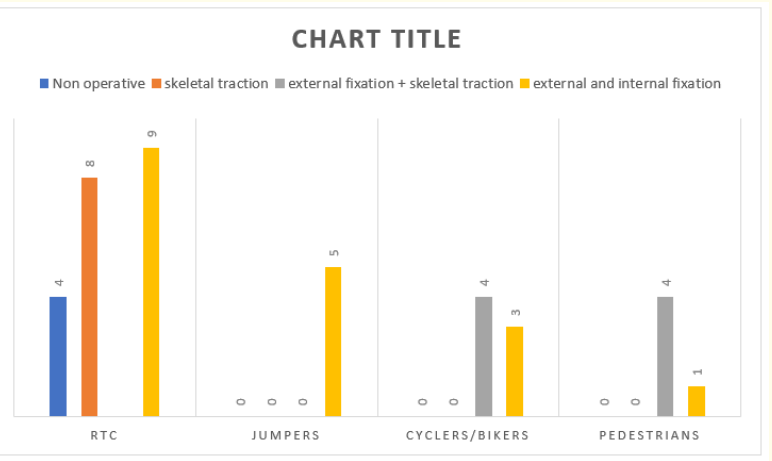

Chart 12: Management in different mechanism of injury in our study has shown that the large group which constitutes RTC has nearly an equal mix of skeletal traction and external/internal fixation while most of the jumpers in our series required surgical intervention patients who had a low velocity accidents like pedestrians were mostly managed conservatively.

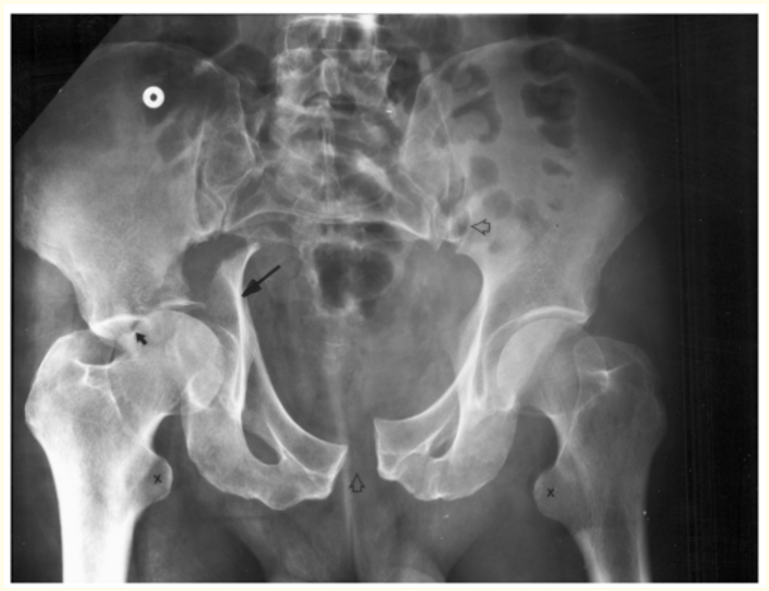

Figure 1: Pelvic $X$ ray in a 40 year old male presenting to A\&E after a RTC showing combined pattern of injury.

and $\mathrm{LC}$ in pelvic ring and AW, AC, T, ABC in acetabulum. Vertical shear [12] and PC, PW acetabular involvement are rare. Combined fractures are often seen with high velocity injuries and more frequently require blood transfusion, ISS in combined fractures is higher than the isolated fractures. I feel We can generally group the fracture into 3 probable pattern or types.

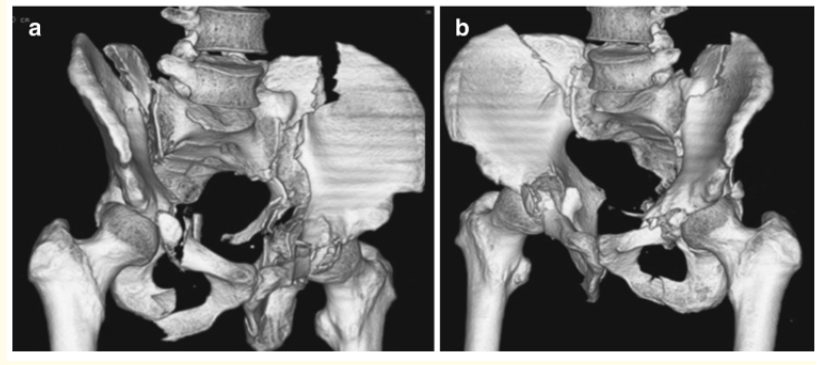

Figure 2: CT Scan for Combined Pelvic and acetabular fractures in a 70 year old following an RTC.

Type 1 fractures: RTC causes fracture involving APC 1, 2, 3 along with $\mathrm{AW}, \mathrm{AC}, \mathrm{ABC}$ variety, lateral compression fractures are rare in RTC, hemodynamic stability is dependent of grade of APC than acetabular fracture pattern and treatment can vary from non-operative to complex surgeries.

Type 2 fractures: Jumpers commonly sustain LC $+\mathrm{ABC}$ pattern and have high ISS when compared to RTC, majority of them are hemodynamically unstable and require blood products and more often end up in a broad spectrum of pelvic surgery. Mortality rate is high, and we found nearly $30 \%$ of them die within 24 hours in our study.

Type 3: Cyclers and pedestrians have an equal incidence of type 1 and type 2 fractures and rarely presents with a combination of type 1 and 2 . ISS, blood transfusion and mortality are like type 1 and type 2 combined fractures and treatment must be designed after a thorough understanding of fracture types. Often require complex surgery.

Although we have proposed the fracture pattern types the complexity of these injuries may require separate treatment strategies. Previous evidence in literature often proved that outcomes of surgical fixation in isolated pelvic ring and acetabular fractures are poor and therefore when dealing with a combined fracture, we suggest seeing these as separate entities and try to achieve anatomic reduction and rigid fixation [32]. Finally, we recommend further studies with large sample size to determine the fracture pattern and measure subjective and objective outcomes of surgical fixation in combined fractures. 


\section{Limitation of Study}

In our retrospective analysis there are inherent limitations. As discussed earlier its not possible to design a randomised control trial to determine our outcomes owing to the rarity of the fractures. An improvement in this study could have been made by including a matched control group [35] but this would cause added up difficulties and leads to selection bias.

Firstly, Sample size of our study is one the most significant limitations as it gives decreased accuracy in measurable observations and our ability to perform multivariate analysis. There is heterogenicity in the study population due to patient factor and injury factors, and the heterogenicity could not be decreased when we subdivided the population into various groups, moreover the injury pattern is not the same in two different patients, some have simple fractures and some have complex and combinations of complex fractures and it can be said when dealing with multiply injured patients there will be heterogenicity as no two injuries are same.

Secondly, A retrospective data collection advances itself to dependency on previously accumulated data and the reported data in our study relies on the people who accumulated it overtime. I have made great efforts to double check the charts from various sources but this was not always the case. A prospectively collected data would have made the study stronger as the primary investigator could ensure the reliability of the data and may be able to collect extra information which is not available in our current pelvic trauma [37] database.

\section{Conclusion}

Classification in orthopaedics has historically been an academic process mostly attributed to the person who has made a major contribution to the topic usually associated with their name or institution these strategy has so far proved to be of limited value in predicting and offering any real value in management of patient injury barring a few usually the good the bad and the ugly aspect of fractures has been recognised radiologically by most of the surgeons who mostly manage the surgery based on the complexity of the injury, Our humble effort in this whole process has been to categorise injuries based on the mechanism of injury combined with radiological parameters [43]. It provides a good value in communicating to other trauma units regarding patient care and management, hope will lead to a better care in management of these complex injuries, that more research and evidence which will be required in the process to further validate and evaluate.
Although we have proposed the fracture pattern types the complexity [49] of these injuries may require separate treatment strategies. Previous evidence in literature often proved that outcomes of surgical fixation in isolated pelvic ring and acetabular fractures are poor and therefore when dealing with a combined fracture, we suggest seeing these as separate entities and try to achieve anatomic reduction and rigid fixation. Finally, we recommend further studies with large sample size to determine the fracture pattern [50] and measure subjective and objective outcomes of surgical fixation in combined fractures.

\section{Bibliography}

1. Beck M., et al. "The acetabular blood supply: Implications for periacetabular osteotomies". Surgical and Radiologic Anatomy 25 (2003): 361-367.

2. Burgess AR., et al. "Pelvic ring disruptions: effective classification system and treatment protocols". The Journal of Trauma 30.7 (1990): 848-856.

3. Cosker TDA., et al. "Pelvic ramus fractures in the elderly: 50 patients studied with MRI". Acta Orthopaedica 76.4 (2005): 513516.

4. Collins H. "Creative research- The theory and practice of Research for the creative industries- AVA publications (2010): 38

5. Dalal SA., et al. "Pelvic fracture in multiple trauma: Classification by mechanism is key to pattern of organ injury, resuscitative requirements, and outcome". The Journal of Trauma 29.7 (1989): 981-1000.

6. Errel W., et al. "Control of severe haemorrhage using c clamp and pelvic packing in multiply injured patients with pelvic ring disruption". Journal of Trauma and Orthopaedic 15 (2001): 468.

7. Fox MA., et al. "Pelvic fractures: analysis of factors affecting pre hospital triage and patient outcomes". Southern Medical Journal 83 (1990): 785.

8. Ganz R., et al. "The anti shock pelvic clamp/ clinical application" 167 (1991): 71.

9. Gänsslen A., et al. "Epidemiology of pelvic ring injuries". Injury 27 (1996).

10. Gertzbein SD and Chenoweth DR. "Occult injuries of the pelvic ring". Clinical Orthopaedics 128 (1977): 202-207. 
11. Golden RD., et al. "How much vertical displacement of the symphysis indicates instability after pelvic injury?" The Journal of Trauma and Acute Care Surgery 74.2 (2013): 585-589.

12. Greenwald AS and O'Connor JJ. "The transmission of load through the human hip joint". The Journal of Biomechanics 4 (1971): 507-528.

13. Gray H. "Anatomy of the Human Body. Philadelphia, PA: Lea and Febiger; 1918. Bartleby (2000).

14. Ghanyam AJ., et al. "The effects of laparotomy and external fixator stabilisation on pelvic volume /journal of trauma 38 (1995): 396.

15. Gylling SF., et al. "Immediate external fixator application to unstable pelvic fractures". American Journal of Surgery 150 (1985): 721.

16. Holdsworth FW. "Dislocation and fracture dislocation of the pelvis". Journal of Bone and Joint Surgery 30B (1948): 461.

17. Jason J Halvorson., et al. "Combined acetabulum and pelvic ring injuries". Journal of the American Academy of Orthopaedic Surgeons 22 (2014): 304-314.

18. Judet R., et al. "Fractures of the acetabulum: Classification and surgical approaches for open reduction.Preliminary report". Journal of Bone and Joint Surgery American 46 (1964): 16151646.

19. Köhnlein W., et al. "Acetabular morphology: Implications for joint-preserving surgery". Clinical Orthopaedics and Related Research 467 (2009): 682-691.

20. Letournel E. "Aectabular fractures: Classification and management". Clinical Orthopaedics 151 (1980): 81-123.

21. Magnussen RA., et al. "Predicting blood loss in isolated pelvic and acetabular high-energy trauma". Journal of Orthopaedic Trauma 21.9 (2007): 603-607.

22. Manson TT., et al. "Embolization of pelvic arterial injury is a risk factor for deep infection after acetabular fracturesurgery". Journal of Orthopaedic Trauma 27.1 (2013): 11-15.

23. Gardner MJ and Farrell Matta JM. "Fractures of the acetabulum: Accuracy of reduction clinical results in patients managed operatively within three week after the injury". Journal of Bone and Joint Surgery American 78.11 (1996): 1632-1645.

24. Marsh JL., et al. "Fracture and dislocation classification compendium - 2007: Orthopaedic Trauma Association classification, database and outcomes committee". Journal of Orthopaedic Trauma 21.10 (2007): S1-S133.

25. Mears SC and Berry DJ. "Outcomes of displaced and nondisplaced pelvic and sacral fractures in elderly adults". Journal of the American Geriatrics Society 59.7 (2011): 1309-1312.

26. Oliver CW., et al. "Outcome after pelvic ring fractures: evaluation using the medical outcomes short form SF-36". Injury 27.9 (1996): 635-641.

27. Osgood GM., et al. "Combined pelvic ring disruption and acetabular fracture:Associated injury patterns in 40 patients". Journal of Orthopaedic Trauma 27.5 (2013): 243-247.

28. Plaisier BR., et al. "Improved outcome afterearly fixation of acetabular fractures". Injury 31.2 (2000): 81-84.

29. Pennal GF., et al. "Pelvic disruption: assessment and classification". Clinical Orthopaedics 151 (1980): 12-21.

30. Pohlemann T., et al. "The Hannover experience in management of pelvic fractures”. Clinical Orthopaedics 305 (1994): 69-80.

31. Pohlemann T., et al. "Outcome after pelvic ring injuries". Injury 27.2 (1996): B31-38.

32. Porter SE., et al. "Acetabular fracture patterns and their associated injuries". Journal of Orthopaedic Trauma 22.3 (2008): 165170.

33. Ponseti IV. "Growth and development of the acetabulum in the normal child. Anatomical, histological, and roentgenographic studies". Journal of Bone and Joint Surgery American 60 (1978): 575-585.

34. Prasad A and GG Lloyd. "Attempted suicide by jumping". Acta Psychiatrica Scandinavica 68.5 (1983): 394-396.

35. Rommens PM and Hessmann MH. "Staged reconstruction of pelvic ring disruption: Differences in morbidity, mortality,radiologic results, and functional outcomes between B1, B2/B3, and C-type lesions". Journal of Orthopaedic Trauma 16.2 (2002): 92-98. 
36. Richardson J., et al. "Open pelvic fracture". The Journal of Trauma 22 (1982): 533.

37. Simonian PT., et al. "Biomechanical simulation of the anteroposterior compression injury of the pelvis. An understanding of instability and fixation". Clinical Orthopaedics 309 (1994): 245-256.

38. Stover MD., et al. "Pelvic Ring Disruptions. In: Skeletal Trauma: Basic Science, Management and Reconstruction". Fourth. Saunders Elsevier (2009): 1107-1169.

39. Suzuki T., et al. "Longterm functional outcome after unstable pelvic ring fracture". The Journal of Trauma 63.4 (2007): 884888.

40. Suzuki T., et al. "Combined injuries of the pelvis and acetabulum: Nature of a devastating dyad". Journal of Orthopaedic Trauma 24.5 (2010): 303-308.

41. The J., et al. "Jumpers and fallers: a comparison of the distribution of skeletal injury". Clinical Radiology 58.6 (2003): 482-486.

42. Tibbs BM., et al. "DVAcetabular and isolated pelvic ring fractures: A comparison of initial assessment and outcome". The American Surgeon: SAGE Journals 74.6 (2008): 538-541.

43. Tile M. "Pelvic ring fractures: Should they be fixed?" The Journal of Bone and Joint Surgery British 70.1 (1988): 1-12.

44. Tile M. "Acute Pelvic Fractures: I. Causation and Classification". Journal of the American Academy of Orthopaedic Surgeons 4.3 (1996): 143-151.

45. Tile M and Olson S. "Decision making: Nonoperative and operative indications for acetabular fractures". In Tile M, Helfet DL, Kellam : Fractures of the Pelvis and Acetabulum, ed 3. Philadelphia, PA, Lippincott Williams and Wilkins (2003): 496-532.

46. Thompson JC and Netter FH. "Netter's Concise Orthopaedic Anatomy". 2nd edition. Philadelphia, PA: Saunders Elsevier (2010).

47. Young JW., et al. "Lateral compression fractures of the pelvis: the importance of plain radiographs in the diagnosis and surgical management”. Skeletal Radiology 15.2 (1986):103109.

48. Young JW., et al. "Pelvic fractures: value of plain radiography in early assessment and management Radiology 160.2 (1986): 445-451.
49. Wasielewski LA., et al. "Acetabulum anatomy and transacetabular screw fixation in total hip arthroplasty". Journal of Bone and Joint Surgery American 72 (1990): 501-508.

50. Westerborn A. "Beitrage zur kenntniss der Beckenbrueche and Beckenluxationen”. Acta Chirurgica Scandinavica 8 (1928).

51. Wilenius R. "Ubher Beckenbrueche". Acta Chirurgica Scandinavica 79 (1973).

52. Wright R., et al. "Acetabular fractures: long-term follow-up of open reduction and internal fixation". Journal of Orthopaedic Trauma 8.5 (1994): 397-403.

\section{Volume 4 Issue 6 June 2021}

(c) All rights are reserved by Senthil Kumar Selvarajan., et al. 